Pacific Journal of Mathematics

APPLICATIONS OF APPROXIMATION THEORY TO
DIFFERENTIAL EQUATIONS WITH DEVIATING ARGUMENTS 


\title{
APPLICATIONS OF APPROXIMATION THEORY TO DIFFERENTIAL EQUATIONS WITH DEVIATING ARGUMENTS
}

\author{
M. S. HENRY AND K. WigGins
}

The authors utilize fundamental approximation theory concepts to establish an existence theorem for an initial value problem with a deviating argument. The techniques used to establish this theorem result in an algorithm to compute polynomial approximations to the solution of the initial value problem.

1. Introduction. In recent years several authors $[2,5,8,9$, $10,12]$ have considered best approximating in some sense the solutions to various types of nonlinear differential and integro-differential equations. These papers have generally been devoted to examining questions involving the existence of best approximations and with proving that appropriate sequences of approximations converge to a solution of the differential or integro-differential equation. The best approximation problems of the above references are ordinarily nonlinear and consequently computational questions are not readily resolved.

More recently Allinger [1], Henry [1, 6] and Wiggins [6] have developed alternative approximation theory approaches that are computationally adaptive.

In this regard [6] deals with nonlinear initial value problems without deviating arguments, whereas [1] primarily considers linear initial value problems with deviating arguments. The approximation problems of [6] and [1] are basically different. The goal of the present paper is to extend the results of [6] to arbitrary order and to the more difficult deviating argument case. The theory developed in the present paper will not contain the linear theory of [1]; in a subsequent paper [7] the authors will extend the results in [1] and relate those extensions to the fundamental results of the present paper.

2. The initial value problem. Consider the scalar initial value problem

$$
\begin{aligned}
& x^{(n)}(t)-\hat{f}\left(t, x(t), \cdots, x^{(n-1)}(t), x(h(t)), \cdots, x^{(n-1)}(h(t))\right)=0, \\
& x^{(i)}(0)=\alpha_{i}, i=0,1, \cdots, n-1, t \in J=[-\gamma, \tau],
\end{aligned}
$$

where $\gamma \geqq 0, \tau \geqq 0$, and $\gamma+\tau>0$. This initial value problem is 
considered in [6] for $h(t)=t$ and $n=2$. A function $x$ is a solution to initial value problem $(I V P)(1)$ on $J$ if the $x^{(i)}(t), i=0, \cdots, n$ are defined for $t \in J \cup h(J)$ and if $x$ satisfies $I V P$ (1) for $t \in J$. In (1) it is assumed that $\hat{f}: J \times R^{2 n} \rightarrow R$ is continuous, and that $h \in$ $C(J)$. Other hypotheses will be imposed later. We note that (1) generalizes not only the initial value problem considered in [6], but also the initial value problem with deviating argument of [1].

From a theoretical viewpoint, IVP (1) can be examined more efficiently in an equivalent vector setting. The scalar form will be utilized in the computations.

The standard transformation

$$
X(t)=\left[x_{1}(t), \cdots, x_{n}(t)\right]^{T}=\left[x(t), \cdots, x^{(n-1)}(t)\right]^{T}
$$

converts $I V P(1)$ into a companion vector $I V P$

$$
\begin{aligned}
& \dot{X}(t)=\bar{A} X(t)+F(t, X(t), X(h(t))), \\
& X(0)=\Lambda, \quad t \in J,
\end{aligned}
$$

where $\Lambda=\left(\alpha_{0}, \cdots, \alpha_{n-1}\right)^{T}$,

$$
A=\left(\begin{array}{cccccc}
0 & 1 & 0 & \cdots & 0 & 0 \\
0 & 0 & 1 & \cdots & 0 & 0 \\
0 & 0 & 0 & \cdots & 0 & 1 \\
0 & 0 & 0 & \cdots & 0 & 0
\end{array}\right),
$$

and

$$
F(t, X(t), X(h(t)))=\left[0, \cdots, 0, f_{h}[x](t)\right]^{T},
$$

where for notational convenience

$$
f_{h}[x](t)=\hat{f}\left(t, x(t), \cdots, x^{(n-1)}(t), x(h(t)), \cdots, x^{(n-1)}(h(t))\right) .
$$

The conditions on $\hat{f}$ imply that $F: J \times R^{2 n} \rightarrow R^{n}$ may be assumed to be continuous. Let $\Pi_{k}$ denote the set of scalar polynomials of degree at most $k$. Define

$$
\begin{aligned}
\mathscr{P}_{k}= & \left\{p \in \Pi_{k}: p(t)=c_{0}+c_{1} t+\cdots+c_{n-1} t^{n-1}+b_{n} t^{n}\right. \\
& +\cdots+b_{k} t^{t}, t \in J, \text { where } c_{i}=\alpha_{i} / i ! \text { and } \\
& \left.b_{i} \in R, \quad(i=n, n+1, \cdots, k)\right\} .
\end{aligned}
$$

Thus $\mathscr{P}_{k}$ is the set of all scalar polynomials of degree at most $k$ that satisfy the initial conditions of (1). Let $\hat{\Pi}_{k}$ denote the set of functions $P: J \rightarrow R^{n}$ such that each component of $P$ is an element of $\Pi_{k}$. Corresponding to (5), define 


$$
\widehat{\mathscr{P}}_{k}=\left\{P \in \hat{\Pi}_{k}: P=\left[p, \dot{p}, \cdots, p^{(n-1)}\right]^{T}, \quad p \in \mathscr{P}_{k}\right\} .
$$

Hence for each $P \in \hat{\mathscr{P}}_{k}, P(0)=\Lambda$. Let $\bar{Z}(t)$ be the fundamental matrix solution to the matrix initial value problem

$$
\dot{\bar{X}}(t)=\bar{A} \bar{X}(t), \bar{X}(0)=I, t \in J .
$$

Hereafter, for $X=\left[x_{1}, \cdots, x_{n}\right] \in R^{n}$,

$$
|X|=\max _{1 \leqq i \leqq n}\left(\left|x_{i}\right|\right)
$$

if $X$ is a continuous mapping from $J$ to $R^{n}$, then

$$
|X|_{J}=\max _{J} \max _{1 \leqq i \leqq n}\left(\left|x_{i}(t)\right|\right) \text {. }
$$

Because of the nature of the matrix $\bar{A}$, the solution $\bar{Z} A$ to the vector initial value problem

$$
\dot{X}(t)=\bar{A} X(t), \quad X(0)=\Lambda, \quad t \in J,
$$

is an element of $\hat{\mathscr{P}}_{k}$. Due to the continuity of $F$, there exists a positive constant $M$ depending on $N$ such that $|F(t, X, Y)| \leqq M$ for all $(t, X, Y) \in J \times R^{2 n}$ with $\max (|X|,|Y|) \leqq N$. Let $\bar{Z}(t)=\left(z_{i j}(t)\right)$, $t \in J$. Define

$$
\alpha=|\bar{Z}|_{J}=\max _{J} \max _{1 \leqq i \leqq n}\left(\sum_{j=1}^{n}\left|z_{i j}(t)\right|\right) .
$$

Then $J_{1}=\left[-\gamma_{1}, \tau_{1}\right] \subseteq J, \gamma_{1}$ and $\tau_{1}$ nonnegative with $\gamma_{1}+\tau_{1}>0$, and an appropriate deviating argument $g$ are determined as follows:

$H_{1}$. Let $N \geqq 1+|\bar{Z} \Lambda|_{J}$. Choose $J_{1}$ to insure that for all $t \in J_{1}$, $2|t| M \alpha \leqq 1$.

$H_{2}$. Assume an interval $J_{2}=\left[-\gamma_{2}, \tau_{2}\right], \gamma_{2}$ and $\tau_{2}$ nonnegative with $\gamma_{2}+\tau_{2}>0$, satisfying $J_{2} \leqq J_{1}$ and $h\left(J_{2}\right) \leqq J_{1}$, exists. Define $g \in C\left(J_{1}\right)$ by $g(t)=h(t), t \in J_{2}, g\left(J_{1}\right) \subseteq J_{1}$.

REMARK. If $h(0)=0$, a condition frequently required of the deviating argument (see [11]), then the existence of a $J_{2}$ and $g$ satisfying $\mathrm{H}_{2}$ is assured.

Assuming hypotheses $H_{1}$ and $H_{2}$, an alternative to initial value problem (1A) is

$$
\begin{aligned}
& \dot{X}(t)=\bar{A} X(t)+F(t, X(t), X(g(t))), \\
& X(0)=\Lambda, \quad t \in J_{1} .
\end{aligned}
$$


3. An existence theorem. We are now in a position to define an approximating scheme that results in a constructive existence theorem for $\operatorname{IVP}(1 \mathrm{~B})$ and hence for $(1 \mathrm{~A})$ and (1).

Let

$$
S_{k}=\left\{P \in \widehat{\mathscr{P}}_{k}:|P-\bar{Z} \Lambda| \leqq 2|t| M \alpha, \quad t \in J_{1}\right\} .
$$

Then $S_{k}$ is a compact, convex subset of $\hat{\mathscr{P}}_{k}$. For $x \in C^{n-1}\left(J_{1}\right)$, let

$$
\hat{F}[X](t)=F(t, X(t), X(g(t))),
$$

where again $X(t)$ is given by (2). If $P \in S_{k}$, consider the best approximation problem

$$
\inf _{\substack{V \in \hat{\mathscr{V}}_{k}}}|\dot{V}-\bar{A} V-\hat{F}[P]|_{J_{1}}
$$

Because of the nature of $\bar{A}$ and $\hat{\mathscr{P}}_{k}$, this minimization problem reduces to the scalar best approximation problem

$$
\inf _{v \in I_{k-n}}\|v-f[p]\|_{J_{1}},
$$

where $p$ is the first component of $P$ and where $f[p]=f_{g}[p]$. Since $f: C^{n-1}\left(J_{1}\right) \rightarrow C\left(J_{1}\right)$, it is well known from classical approximation theory that there exists a $\bar{v} \in \Pi_{n-k}$ such that

$$
\inf _{v \in \Pi_{k-n}}\|v-f[p]\|_{J_{1}}=\|\bar{v}-f[p]\|_{J_{1}} .
$$

Integrate $\bar{v} n$ times to obtain the $k$ th degree polynomial $v_{b} \in \mathscr{P}_{k}$. If $V_{b}=\left(v_{b}, \cdots, v_{b}^{(n-1)}\right)$, then

$$
\inf _{\nabla \in \hat{\mathscr{G}}_{k}}|\dot{V}-\bar{A} V-\hat{F}[P]|_{J_{1}}=\left|\dot{V}_{b}-\bar{A} V_{b}-\hat{F}[P]\right|_{J_{1}} \text {. }
$$

Define an operator $T_{k}$ on $S_{k}$ as follows: if $P \in S_{k}$, then

$$
T_{k}[P](t)=V_{b}(t), \quad t \in J_{1} .
$$

Thus if $P \in S_{k}, T_{k}[P]$ is the best approximation from $\hat{\mathscr{P}}_{k}$ to $\hat{F}[P]$ in the sense of (14). Note that since (13) has a unique solution $v_{b}$, $V_{b}$ is also unique.

THEOREM 1. Assume that $\hat{f}$ and $h$ satisfy the continuity requirements described below (1), and that $H_{1}$ and $H_{2}$ are valid. Then $T_{k}$ is a continuous mapping from $S_{k}$ into $S_{k}$.

Proof. Suppose that $T_{k}[P]=V_{b}$. Let

$$
\dot{V}_{b}(t)-\bar{A} V_{b}(t)-\hat{F}[P](t)=E(t) .
$$


Since $P \in S_{k}, H_{1}$ and $H_{2}$ imply that $|\hat{F}[P]|_{J_{1}} \leqq M$, and consequently, since $\bar{Z}(t) \Lambda \in \widehat{\mathscr{P}}_{k}$ and $V_{b}$ is best in the sense of (14),

$$
\begin{aligned}
& \left|\dot{V}_{b}-\bar{A} V_{b}-\hat{F}[P]\right|_{J_{1}} \\
& \quad \leqq|\dot{\bar{Z}} \Lambda-\bar{A} \bar{Z} \Lambda-\hat{F}[P]|_{J_{1}} \\
& \quad=|\hat{F}[P]|_{J_{1}} \leqq M .
\end{aligned}
$$

This inequality and (16) imply that

$$
|E|_{J_{1}} \leqq M
$$

Now (16) holds if and only if

$$
\begin{aligned}
V_{b}(t)- & \bar{Z}(t) \Lambda \\
= & \int_{0}^{t} \bar{Z}(t) \bar{Z}^{-1}(s) \hat{F}[P](s) d s \\
& +\int_{0}^{t} \bar{Z}(t) \bar{Z}^{-1}(s) E(s) d s .
\end{aligned}
$$

A variation of parameters argument readily verifies equality (18). Since $\bar{A}$ is a constant matrix, $\bar{Z}(t) \bar{Z}^{-1}(s)=\bar{Z}(t-s)$; therefore (17) and (18) imply that

$$
\begin{aligned}
\left|V_{b}-\bar{Z} \Lambda\right| \leqq 2|t| M|\bar{Z}|_{J_{1}} \\
=2|t| M \alpha, \quad t \in J_{1} .
\end{aligned}
$$

Thus $V_{b} \in S_{k}$ and hence $T_{k}$ maps $S_{k}$ into $S_{k}$.

Establishing that $T_{k}$ is continuous is somewhat more difficult and requires the use of fundamental concepts of approximation theory. Suppose that $P \in S_{k}$ and that $\bar{P}$ is any other element of $S_{k}$; if $T_{k}[P]=Q$ and $T_{k}[\bar{P}]=\bar{Q}$, then as in (13),

$$
\inf _{v \in \Pi_{k-n}}\|v-f[p]\|_{J_{1}}=\left\|q^{(n)}-f[p]\right\|_{J_{1}}
$$

and

$$
\inf _{v \in \Pi_{k-n}}\|v-f[\bar{p}]\|_{J_{1}}=\left\|\bar{q}^{(n)}-f[\bar{p}]\right\|,
$$

where $p, \bar{p}, q$, and $\bar{q}$ are the first components of $P, \bar{P}, Q$, and $\bar{Q}$, respectively. The classical Freud theorem [3, p. 82] of approximation theory implies that

$$
\left\|q^{(n)}-\bar{q}^{(n)}\right\|_{J_{1}} \leqq \lambda_{p}\|f[p]-f[\bar{p}]\|_{J_{1}} .
$$

Let $\varepsilon>0$ be given. Since $\hat{f}$ is uniformly continuous on compact subsets of $J \times R^{2 n}$, there exists a $\delta>0$ such that if $|P-\bar{P}|_{J_{1}}<\delta$, 


$$
\begin{gathered}
\|f[p]-f[\bar{p}]\|_{J_{1}}<\frac{\varepsilon}{\lambda_{p}} \frac{1}{\max _{0 \leqq i \geqq n}\left[\max \left(\gamma_{1}, \tau_{1}\right)\right]^{n-i}}, \\
i=0,1, \cdots, n .
\end{gathered}
$$

Inequalities (19) and (20) imply that

$$
\left|q^{(i)}(t)-\bar{q}^{(i)}(t)\right|<\varepsilon \quad i=0,1, \cdots, n-1, t \in J .
$$

Thus

$$
|Q(t)-\bar{Q}(t)|<\varepsilon .
$$

Since $T_{k}[P]=Q$ and $T_{k}[\bar{P}]=\bar{Q}$, the above analysis establishes that given $P \in S_{k}$ and $\varepsilon>0$, there exists $\delta_{o}>0$ such that if $\bar{P} \in S_{k}$ and $|P-\bar{P}|_{J_{1}}<\delta$, then

$$
\left|T_{k}[P]-T_{k}[\bar{P}]\right|_{J_{1}}<\varepsilon .
$$

Hence $T_{k}$ is a continuous mapping from $S_{k}$ into $S_{k}$ and the proof is complete.

CoROLLARY 1. Assume the hypotheses of Theorem 1 are valid. Then the mapping $T_{k}$ has a fixed point in $S_{k}$.

Proof. Since $S_{k}$ is a compact, convex subset of $\hat{\mathscr{P}}_{k}$, the result follows from the Schauder fixed point theorem [16, p. 25].

We summarize the results of Theorem 1 and Corollary 1. There exists a $P \in \widehat{\mathscr{P}}_{k}$ such that if we seek the best approximation $V_{b}$ to $\hat{F}[P]$ in the sense of (14), this best approximation is precisely $V_{b}=$ $P$. In the equivalent scalar form, if we seek the best approximation $\bar{v}$ from $\Pi_{k-n}$ to $f[p]$ in the sense of (13), then $\bar{v}=p^{(n)}$.

The proof of Theorem 1 does not depend on $k$, and consequently the hypotheses of Theorem 1 imply that there exists a sequence

$$
\left\{P_{k}(t)\right\}_{k=n}^{\infty}, \quad t \in J_{1},
$$

of fixed points, one for each operator $T_{k}, k \geqq n$.

Define $E_{k}, k \geqq n$, by

$$
\dot{P}_{k}(t)-\bar{A} P_{k}(t)-\hat{F}\left[P_{k}\right](t)=E_{k}(t),
$$

$t \in J_{1}$, where $P_{k}$ is an element of the sequence (22). Since $P_{k} \in S_{k}$, $k \geqq n$,

$$
\left|P_{k}(t)-\bar{Z}(t) \Lambda\right| \leqq 2|t| M \alpha, \quad t \in J_{1} .
$$

Equation (23) and inequalities (17) and (24) imply that the sequences

$$
\left\{\left|P_{k}\right|_{J_{1}}\right\}_{k=n}^{\infty} \text { and }\left\{\left|\dot{P}_{k}\right|_{J_{1}}\right\}_{k=n}^{\infty}
$$


are bounded. Thus the sequence (22) is an equicontinuous and uniformly bounded family on $J_{1}$. Let $W$ be a cluster point of this sequence. Equality (23) is valid if and only if

$$
\begin{aligned}
P_{k}(t) & -\bar{Z}(t) \Lambda=\int_{0}^{t} \bar{Z}(t) \bar{Z}^{-1}(s) \hat{F}\left[P_{k}\right](s) d s \\
& +\int_{0}^{t} \bar{Z}(t) \bar{Z}^{-1}(s) E_{k}(s) d s .
\end{aligned}
$$

Thus if

$$
\lim _{k \rightarrow \infty}\left|E_{k}\right|_{J_{1}}=0
$$

then (26) implies that $W$ is a solution to $I V P$ (1B). Thus $W$ is a solution to $I V P(1 \mathrm{~A})$ on $J_{2}$, and the first entry $w$ of $W$ satisfies IVP (1) on $J_{2}$. These observations constitute part of the conclusions of the next theorem.

THEOREM 2. Suppose the hypotheses of Theorem 1 are satisfied. Then (27) is valid. If $W$ is a cluster point of (22) on $J_{1}$, then $W$ is a solution of IVP (1B) on $J_{1}$ and $W$ is a solution of IVP (1A) on $J_{2}$.

Proof. Based on the remarks preceeding the statement of Theorem 2, only (27) needs to be established. Let

$$
P_{k}=\left(p_{k}, \cdots, p_{k}^{(n-1)}\right) .
$$

Then since $P_{k}$ is a fixed point of $T_{k}$,

$$
\inf _{v \in \Pi_{k-n}}\left\|v-f\left[p_{k}\right]\right\|_{J_{1}}=\left\|p_{k}^{(n)}-f\left[p_{k}\right]\right\|_{J_{1}} .
$$

If

$$
e_{k-n}\left(f\left[p_{k}\right]\right)=\left\|p_{k}^{(n)}-f\left[p_{k}\right]\right\|_{J_{1}},
$$

then

$$
e_{k-n}\left(f\left[p_{k}\right]\right)=\left|E_{k}\right|_{J_{1}} \text {. }
$$

Jackson's theorem [13, p. 22] implies that

$$
e_{k-n}\left(f\left[p_{k}\right]\right) \leqq 6 \omega_{k}\left(\frac{L}{k-n}\right)
$$

where $L$ is half the length of the interval $J_{1}$, and $\omega_{k}$ is the modulus of continuity of $f\left[p_{k}\right]$ on $J_{1}$. Let $\varepsilon>0$ be given. Since the sequences (25) are bounded, the sequences

$$
\left\{p_{k}^{(i)}\right\}_{k=n}^{\infty}, \quad i=0, \cdots, n-1,
$$


are uniformly bounded and equicontinuous families on $J_{1}$. Thus the uniform continuity of $\hat{f}$ on compact subsets of $J_{1} \times R^{2 n}$ implies that the sequence $\left\{f\left[p_{k}\right]\right\}_{k=n}^{\infty}$ is an equicontinuous family on $J_{1}$. Consequently given $\varepsilon>0$ there exists a $\delta>0$ such that $|t-s| \leqq \delta$ implies that $\left|f\left[p_{k}\right](t)-f\left[p_{k}\right](s)\right| \leqq \varepsilon$, independent of $k$. Therefore $\omega_{k}(\delta) \leqq \varepsilon$, independent of $k$. If $K$ is large enough to insure that for all $k \geqq K, L /(k-n) \leqq \delta$, then the monotonicity of $\omega_{k}[13, \mathrm{p} .14]$ implies for all $k \geqq K$,

$$
\omega_{k}\left(\frac{L}{k-n}\right) \leqq \omega_{k}(\delta) \leqq \varepsilon .
$$

Thus for all $k \geqq K, \quad(29)$ and (30) imply that $\left|E_{k}\right|_{J_{1}} \leqq 6 \varepsilon$. Consequently $\lim _{k \rightarrow \infty}\left|E_{k}\right|_{J_{1}}=0$, completing the proof.

4. Computations. We now describe an algorithm based on the theory of section three. For $k$ sufficiently large, initially choose $P_{k 0}(t)=\bar{Z}(t) \Lambda$. At the $l+1$ step solve via the second algorithm of Remes [3, p. 97] the best approximation problem

$$
\inf _{V \in \mathscr{V} k}\left|\dot{V}-\bar{A} V-\hat{F}\left[P_{k, l}\right]\right|_{J_{1}}=\inf _{v \in \Pi_{k-n}}\left\|v-f\left[p_{k, l}\right]\right\|_{J_{1}} .
$$

Let the solution of this minimizing problem be $P_{k, l+1}$ and continue. We again note that $p_{k, l+1}^{(n)}$ is then the minimizing polynomial to the scalar problem in (31). This procedure yields a unique sequence

$$
\left\{P_{k, l}\right\}_{l=0}^{\infty}
$$

with a corresponding sequence of first elements of (32),

$$
\left\{p_{k, l}\right\}_{l=0}^{\infty} \cdot
$$

The sequences (32) and (33) have cluster points $P_{k}$ and $p_{k}$, respectively, which are approximate solutions of degree $k$ to (1A) and (1) on $J_{2}$.

The actual calculations are carried out in the scalar setting. Furthermore, the computations are effected for any interval $J_{1} \subseteq J$ for which $h\left(J_{1}\right) \cong J_{1}$ If no such interval exists, the construction of an appropriate $g$ would be necessary (see $H_{2}$ ). If convergence does not occur, then $J_{1}$ is reduced (see $H_{1}$ ).

We note that a cluster point of (32) need not be a fixed point of $T_{k}$ in the sense of Corollary 1. However, an additional checkable condition insures that a cluster point of (32) is a fixed point of $T_{k}$. In particular, entries of sequence (33) satisfy

$$
\inf _{v \in \Pi_{k-n}}\left\|v-f\left[p_{k, l}\right]\right\|_{J_{1}}=\left\|p_{k, l+1}^{(n)}-f\left[p_{k, l}\right]\right\|_{J_{1}} .
$$


In calculating $p_{k, l+1}$, an alternating set (see [3, p. 75]) consisting of $k-n+2$ arises. If $X_{l}=\left\{t_{1, l}, \cdots, t_{k-n+2, l}\right\}$ is an alternating set, and if

$$
e_{k, l+1}(t)=p_{k, l+1}^{(n)}(t)-f\left[p_{k, l}\right](t) \text {, }
$$

then

$$
\begin{gathered}
e_{k, l+1}\left(t_{i, l}\right)=-e_{k, l+1}\left(t_{i+1, l}\right), \\
i=1, \cdots, n-k+1 .
\end{gathered}
$$

Let $X=\left\{t_{1}, \cdots, t_{n-k+2}\right\}$ be a cluster point of $\left\{X_{l}\right\}_{l=0}^{\infty}$, and let

$$
e_{k}(t)=p_{k}^{(n)}(t)-f\left[p_{k}\right](t)
$$

where $p_{k}$ is the first entry of the cluster point $P_{k}$ of (32) (and hence a cluster point of (33)). Without loss of generality we can assume that the subsequences of $\left\{X_{l}\right\}$ and of (33) that converge to $X$ and $p_{k}$, respectively, involve the same subscripts.

$H_{3}$. Suppose for $t_{i}, t_{i+1} \in X$ that $e_{k}\left(t_{i}\right)=-e_{k}\left(t_{i+1}\right), i=1, \cdots, k-n+1$, where $e_{k}$ is defined in (34).

We note that this condition is weaker than assuming the alternation theorem.

Assuming $H_{3}$ is valid, $P_{k}$ is a fixed point of $T_{k}$, and consequently the theory of $\S 3$ applies. If (33) actually converges, $H_{3}$ is satisfied.

The following examples employ the algorithm described above. A Fortran IV program using double precision arithmetic is utilized in the calculations. The calculations 'for Examples 1 and 2 were executed on the Xerox Sigma 7 computer, located at Montana State University. The calculations for Example 3 were effected on the IBM 370 , at The College of Charleston.

EXAMPLe 1.

$$
\begin{aligned}
& \ddot{x}(t)-2(2-t) \dot{x}(t) x^{2}(t)=0, \quad J=[-.7, .7] \\
& x(0)=1 / 2, \quad \dot{x}(0)=1 / 4
\end{aligned}
$$

The algorithm is employed over all of $J$, since $g(t)=t$. For $k=5$ approximation is from

$$
\mathscr{P}_{5}=\left\{p(t): p(t)=.5+.25 t+b_{2} t^{2}+b_{3} t^{3}+b_{4} t^{4}+b_{5} t^{5}\right\} .
$$

Let $B_{l}=\left(.5, .25, b_{2 l}, b_{3 l}, b_{4 l}, b_{5 l}\right)$ be the coefficients of the $l$-th iterate $p_{k, l}$ of sequence (33). Then

$$
\begin{aligned}
& B_{0}=(.5, .25,0,0,0,0) \\
& B_{1}=(.5, .25, .125000, .020833,-.005208,-.001562) \\
& B_{15}=(.5, .25, .119879, .057090, .042496, .023350)
\end{aligned}
$$


If the algorithm is terminated with $B_{15}$ (a stopping criteria is employed here), then the cluster point of (33) is

$$
p_{5}(t)=.5+.25 t+.119879 t^{2}+.570909 t^{3}+.424964 t^{4}+.233505 t^{5} .
$$

The actual solution is $x(t)=1 /(2-t)$, and $\left\|x-p_{5}\right\|_{J}=.001779$.

EXAMPLE 2.

$$
\begin{aligned}
& \ddot{x}(t)+4 x(t / 2) \dot{x}(t / 2)=0, J=[-1,1], \\
& x(0)=0, \quad \dot{x}(0)=2 .
\end{aligned}
$$

We observe that $h(J) \subseteq J$. Approximation is again from $\mathscr{P}_{5}$, and

$$
\begin{aligned}
& B_{0}=(0,2,0,0,0,0), \\
& B_{9}=(0,2,0,-\dot{1} .290025,0, .204262) .
\end{aligned}
$$

Then $B_{9}$ and $B_{10}$ are equal through six decimal places. Thus

$$
p_{5}(t)=2 t-1.290025 t^{3}+.204262 t^{5} .
$$

The actual solution is $x(t)=\sin 2 t$ and $\left\|x-p_{5}\right\|_{J}=.006865$.

EXAMPLE 3. $h(t)=1 / 2\left(e^{t}-1\right)$

$$
\begin{aligned}
& \ddot{x}(t)+4[2 x(h(t))+1]^{-2}=0, \quad t \in[-.175,1] \\
& x(0)=0, \quad \dot{x}(0)=2
\end{aligned}
$$

In this example $h(J)=[-.08, .859] \subseteq J$. For approximation from $\mathscr{P}_{7}$, the alternating set is

$$
X=\{-.1750,-.1211, .0509, .3313, .6519, .9054,1.0000\} \text {. }
$$

The actual solution is $x(t)=\ln (2 t+1)$, and

$$
\begin{aligned}
p_{7}(t) & =2 t-1.9688 t^{2}+2.8151 t^{3}-4.7085 t^{4} \\
& +.58950 t^{5}-4.0266 t^{6}+1.0974 t^{7}
\end{aligned}
$$

Hypothesis $H_{3}$ is satisfied by $e_{7}$, and $\left\|e_{7}\right\|=.08519$. Finally, $\| x-$ $p_{7} \|=.00496$.

5. Conclusions. This paper utilizes approximation theory techniques to obtain uniform approximate solutions to variants of $I V P$ (1). The primary result of the paper is an existence theorem for IVP (1). An algorithm based on this existence theorem is used to compute approximate solutions to $I V P(1)$. This paper extends results of the authors [6] to higher order initial value problems 
with deviating arguments. The present paper is closely related to [1], but results in an approximation problem and a companion algorithm that differ from those given in [1] (for additional literature involving other deviating argument problems, see the references of [1]). In a subsequent paper [7] the authors intend to present a theory that parallels the theory of the present paper but contains the linear theory of [1]. The results of the present paper do substantially improve the nonlinear results contained in [1].

\section{REFERENCES}

1. G. Allinger and M.S. Henry, Approximate solutions of differential equations with deviating arguments, SIAM J. Nu. Anal., 13 (1976), 412-426.

2. A. Bacopoulos and A.G. Kartsatos, On polynomials approximating solutions of nonlinear differential equations, Pacific J. Math., 40 (1972), 1-5.

3. E.W. Cheney, Introduction to Approximation Theory, McGraw-Hill, New York, 1966.

4. J. Hale, Functional Differential Equations, Applied Mathematical Sciences, Vol.

I. Springer-Verlag, New York, 1971.

5. M.S. Henry, Best approximate solutions of nonlinear differential equations, J. Approximation Theory, 3 (1970), 59-65.

6. M.S. Henry and K. Wiggins, Applications of approximation theory to the initial value problem, J. Approximation Theory, 17 (1976), 66-85.

7. - A comparison of approximation theory techniques for linear and nonlinear differential equations with deviating arguments, in preparation.

8. R.G. Huffstutler and F.M. Stein, The approximate solution of certain nonlinear differential equations, Proc. Amer. Math. Soc., 19 (1968), 998-1002.

9. The approximate solution of $\dot{y}=F(x, y)$, Pacific J. Math., 24 (1968), 283-289.

10. A.G. Kartsatos and E.B. Saff, Hyperpolynomial approximation of solutions of nonlinear integro-differential equations, Pacific J. Math., 49 (1973), 117-125.

11. R.J. Oberg, On the local existence of solutions of certain functional differential equations, Proc. Amer. Math. Soc., 20 (1969), 295-302.

12. A.G. Petsoulas, The approximate solution of Volterra integral equations, J. Approximation Theory, 14 (1975), 152-159.

13. T.J. Rivlin, An Introduction to the Approximation of Functions, Blaisdell, Waltham, Massachusetts, 1969.

14. G.H. Ryder, Solutions of functional differential equations, Amer. Math. Monthly, 76 (1969), 1031-1033.

15. K. Schmitt, ed., Delay and Functional Differential Equations and Their Applications, Academic Press, New York, 1972.

16. D.R. Smart, Fixed Point Theorems, Cambridge University Press, New York, 1974.

Received May 26, 1977. The first author was partially supported by NSF Grant No. $76-06553$.

Montana State University

BOZEMAN, MT 59715

AND

The College of Charleston 



\section{PACIFIC JOURNAL OF MATHEMATICS}

\section{EDITORS}

RICHARD ARENS (Managing Editor)

University of California

Los Angeles, CA 90024

Charles W. Curtis

University of Oregon

Eugene, OR 97403

C. C. MOORE

University of California

Berkeley, CA 94720

\section{J. DugundJI}

Department of Mathematics

University of Southern California

Los Angeles, CA 90007

R. FinN and J. Milgram

Stanford University

Stanford, CA 94305

\section{ASSOCIATE EDITORS}
E. F. BECKENBACH
B. H. NeumanN
F. WOLF
K. YosHIDA

\section{SUPPORTING INSTITUTIONS}

\author{
UNIVERSITY OF BRITISH COLUMBIA \\ CALIFORNIA INSTITUTE OF TECHNOLOGY \\ UNIVERSITY OF CALIFORNIA \\ MONTANA STATE UNIVERSITY \\ UNIVERSITY OF NEVADA, RENO \\ NEW MEXICO STATE UNIVERSITY \\ OREGON STATE UNIVERSITY \\ UNIVERSITY OF OREGON
}

\author{
UNIVERSITY OF SOUTHERN CALIFORNIA \\ STANFORD UNIVERSITY \\ UNIVERSITY OF HAWAII \\ UNIVERSITY OF TOKYO \\ UNIVERSITY OF UTAH \\ WASHINGTON STATE UNIVERSITY \\ UNIVERSITY OF WASHINGTON
}

The Supporting Institutions listed above contribute to the cost of publication of this Journal, but they are not owners or publishers and have no responsibility for its content or policies.

Mathematical papers intended for publication in the Pacific Journal of Mathematics should be in typed form or offset-reproduced, (not dittoed), double spaced with large margins. Please do not use built up fractions in the text of the manuscript. However, you may use them in the displayed equations. Underline Greek letters in red, German in green, and script in blue. The first paragraph or two must be capable of being used separately as a synopsis of the entire paper. Items of the bibliography should not be cited there unless absolutely necessary, in which case they must be identified by author and journal, rather than by item number. Manuscripts, in triplicate, may be sent to any one of the editors. Please classify according to the scheme of Math. Reviews, Index to Vol. 39. All other communications should be addressed to the managing editor, or Elaine Barth, University of California, Los Angeles, California, 90024.

50 reprints to each author are provided free for each article, only if page charges have been substantially paid. Additional copies may be obtained at cost in multiples of 50 .

The Pacific Journal of Mathematics is issued monthly as of January 1966. Regular subscription rate: $\$ 72.00$ a year (6 Vols., 12 issues). Special rate: $\$ 36.00$ a year to individual members of supporting institutions.

Subscriptions, orders for numbers issued in the last three calendar years, and changes of address should be sent to Pacific Journal of Mathematics, 103 Highland Boulevard, Berkeley, California, 94708. Older back numbers obtainable from Kraus Periodicals Co., Route 100, Millwood, NY 10546.

PUBLISHED BY PACIFIC JOURNAL OF MATHEMATICS, A NON-PROFIT CORPORATION

Printed at Kokusai Bunken Insatsusha (International Academic Printing Co., Ltd.). 8-8, 3-chome, Takadanobaba, Shinjuku-ku, Tokyo 160, Japan.

Copyright (C) 1978 by Pacific Journal of Mathematics Manufactured and first issued in Japan 


\section{Pacific Journal of Mathematics}

\section{Vol. 76, No. $2 \quad$ December, 1978}

Stephanie Brewster Brewer Taylor Alexander, Local and global convexity in complete Riemannian manifolds ...........................

Claudi Alsina i Català, On countable products and algebraic convexifications of probabilistic metric spaces ...............................

Joel David Berman and George Grätzer, Uniform representations of

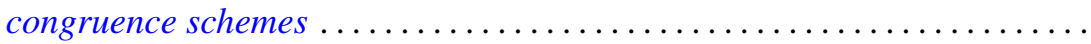

Ajit Kaur Chilana and Kenneth Allen Ross, Spectral synthesis in

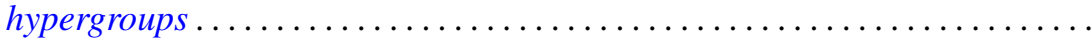

David Mordecai Cohen and Howard Leonard Resnikoff, Hermitian quadratic forms and Hermitian modular forms . .........................

Frank Rimi DeMeyer, Metabelian groups with an irreducible projective

representation of large degree .............................

Robert Ellis, The Furstenberg structure theorem .....................

Heinz W. Engl, Random fixed point theorems for multivalued mappings .......

William Andrew Ettling, On arc length sharpenings ..................

Kent Ralph Fuller and Joel K. Haack, Rings with quivers that are trees........

Kenneth R. Goodearl, Centers of regular self-injective rings ...............

John Gregory, Numerical algorithms for oscillation vectors of second order

differential equations including the Euler-Lagrange equation for

symmetric tridiagonal matrices.

Branko Grünbaum and Geoffrey Shephard, Isotoxal tilings

Myron Stanley Henry and Kenneth Leroy Wiggins, Applications of

approximation theory to differential equations with deviating

arguments

Mark Jungerman, The non-orientable genus of the n-cube .

Robert Richard Kallman, Only trivial Borel measures on $S_{\infty}$ are

quasi-invariant under automorphisms ................

Joyce Longman and Michael Rich, Scalar dependent algebras in the

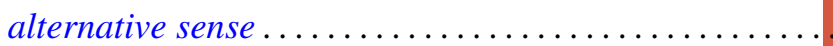

Richard A. Mollin, The Schur group of a field of characteristic zero ........ 471

David Pokrass, Some radical properties of rings with $(a, b, c)=(c, a, b) \ldots 479$

Margaret Shay and Paul Ruel Young, Characterizing the orders changed by

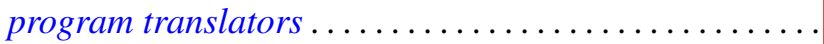

Jerrold Norman Siegel, On the structure of $B_{\infty}(F), F$ a stable space...

Surjeet Singh, (hnp)-rings over which every module admits a basic

submodule...

A. K. Snyder, Universal interpolating sets and the Nevanlinna-Pick property in

Banach spaces of functions...

Jeffrey D. Vaaler, On the metric theory of Diophantine approximation ... 